\title{
Gesture Controlled Robot using Accelerometer
}

\author{
Aishwarya Mohan, Rashmi Priyadarshini
}

\begin{abstract}
Robots are devices that can perform tasks with or without human intervention, based on inputs from its environment. Gestures can be explained as symbolic actions performed by humans having a meaning. In this project, a robot toy car has been developed where the user uses these very gestures to interact with the robot car rather than the established and current technology of using remote controllers with buttons and joysticks, or mobile applications. These gestures are identified with the use of an accelerometer(ADXL345). The entire project is split into two parts: the transmitter circuit and the receiver circuit, where the former uses ADXL345 for gesture recognition, and the latter uses Motor driver (L293D) to move the toy car.
\end{abstract}

Keywords: ADXL345, Arduino SMD, Atmega 328p, Center Shaft DC Motor, L293D, RF Module.

\section{INTRODUCTION}

$\mathrm{R}$ single discipline of human activities. The developments in this field have been going on for decades, however the 21st century particularly has seen immense growth pertaining to this field of technology. Gestures, as stated, can be explained as instinctive actions having a symbolic meaning that are used by a person to express an idea or thought. Robots are electro-mechanical (a portmanteau, reflecting technologies from the disciplines of electronic and mechanical) devices or systems that can perform any tasks without needing any human supervision or else requiring very little of it. These systems perform their tasks based on the logical operations stated in their computer programs that's loaded in their processing unit or microcontroller, taking decisions based on inputs received or read from its environment. Robots primarily fall into two categories: that of being autonomous, and the other being semi-autonomous robots. Autonomous robots are widely used in factories, at industry sites and in manufacturing units as these activities are very high speed operations requiring great accuracy. These kind of robots do not require any human assistance and as the name implies,

Revised Manuscript Received on June 30, 2020.

* Correspondence Author

Aishwarya Mohan*, Department of Electronics and Communication, Sharda University, Greater Noida, India. Email: aishwaryamohan810@gmail.com

Dr. Rashmi Priyadarshini, Department of Electronics and Communication, Sharda University, Greater Noida, India. Email: rashmi.priyadarshini@sharda.ac.in

(C) The Authors. Published by Blue Eyes Intelligence Engineering and Sciences Publication (BEIESP). This is an open access article under the CC BY-NC-ND license (http://creativecommons.org/licenses/by-nc$\underline{\mathrm{nd} / 4.0 / \text { ) }}$ are fully self-sufficient. On the other hand, semi-autonomous robots are used where the decision can not be made by the robots independently, such as in case of tactile controlled, voice controlled and gesture controlled device, where the inputs are touch, audio and movement dependent, thus requiring human assistance in terms of requiring input to facilitate further decision to produce the desired output.

\section{PROBEM STATEMENT}

The innovations in the field of science are currently being developed aggressively in the field of Robotics. It is the rapidly emerging field that is here for the long haul because of its incredible use in rearranging work and day by day errands in the standard existence of people. So as to improve the compatibility of robots with humans for our day to day activities, we have to develop an efficacious method for interacting with robots.

The conventional remote controlled robots turn out to be extremely bulky and it additionally restricts the range of operation of the robots from the remote handling user. For this reason, there have been several advancements in realm of human-machine association.

One normal type of correspondence is motions that are not only constrained to face, body and fingers but in addition include hand signals. This advancement is a huge step ahead so as to move on from the current methods of interaction with devices that are currently in use as remotes, or else tabs and buttons on a mobile application.

So as to expand the utilization of robot in areas where dependency on manpower can be reduced such as in the field of medicine, in operation theatres to move surgery carts, in the automobile assembling industry, in restaurants for serving food to tables, and especially in conditions are not suitable, easily accessible and secure - like rescue operations, surveillance, military activities, and recce patrolling, robots can be created in a manner such as to adhere to the guidelines of its human administrators and follow through with the activity in the instructed manner as ordered. This proposes an incorporated methodology of following and acknowledgment of hand movements which is expected to be utilized as human-robot communication interface.

\section{OBJECTIVE}

The objective of this model is to create a convenient, easy-to-operate human-machine interface for controlling a robot - be it a vehicle, robotic arm, toy or a wheelchair. Our goal is to make this gadget user-friendly and cost-effective so it can be affordable to the general public

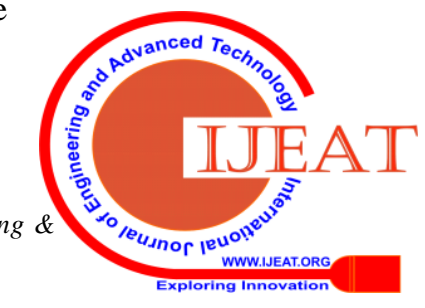




\section{Gesture Controlled Robot using Accelerometer}

and accessible to a big market, along with being utilized for number of purposes. The goal of this undertaking is to construct a vehicle that can be remotely controlled and manipulated by gestures alone, by wearing controller glove and performing predefined motions. The glove is initially designed and programmed with respect to a toy car as object of control. Keeping in mind versatility of its applications, the wearable is programmed to comprehend gestures and function on principle of operation commonly applicable to prospective objects of control, explained in the flowchart Fig. 1.

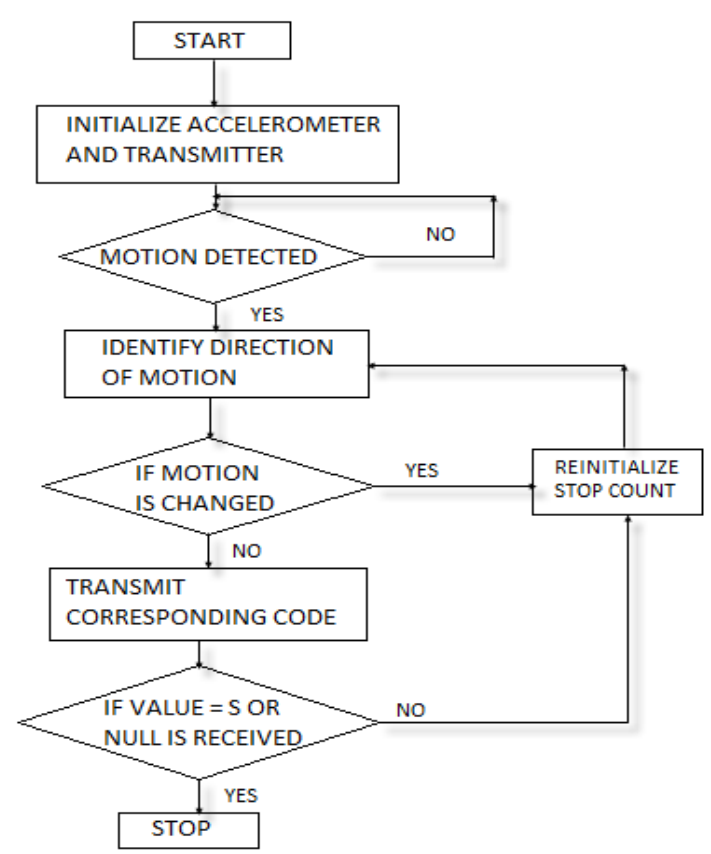

Fig. 1.Principle of operation.

\section{DESCRIPTION OF COMPONENTS}

\section{A. Arduino Uno R3}

Arduino UNO R3 variant uses Atmega328p microcontroller which comprises of different components such as voltage regulator, cation, and crystal oscillator to support the microcontroller. It has 6 analog pins and 14 digital output pins out of which 6 can be used as pulse width modulation (PWM) pins, a barrel jack or a universal serial bus (USB) port can be used to provide power to the board, an in circuit serial programming header [1].

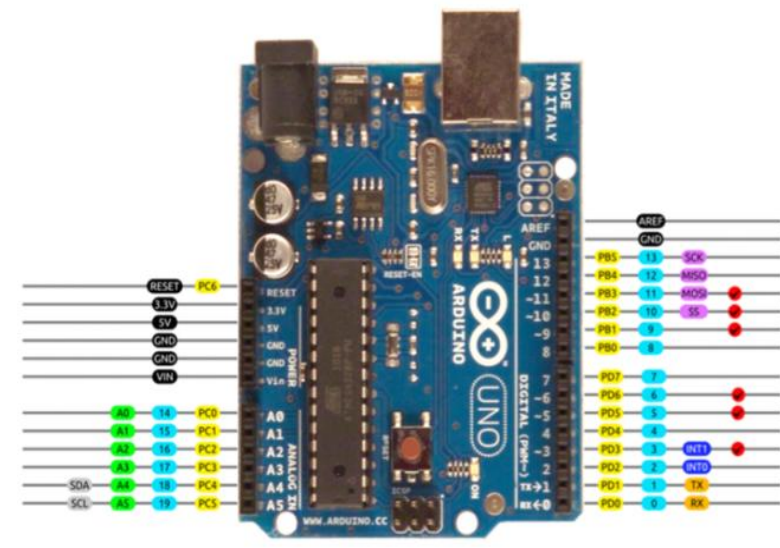

\section{AVR DICITAL ANALOG POWER SERIAL SPI I2C PWM INTERRUPT}

Fig. 2. Arduino Uno R3 board.

\section{B. RF433 Module}

Good The $433 \mathrm{MHz}$ remote module is one of the affordable and simple to use radio frequency (RF) modules for every single remote undertaking. These modules can be utilized only in sets and just simplex communication is plausible, which means the transmitter can just transmit data and the recipient can just receive it, so one can only send information from one point to another in a unidirectional manner [2]. The module has a very limited range of 3 metres but with appropriate antenna and power supply it may be enhanced to a theoretical range of up to 100 metres. Be that as it may, essentially we can scarcely get around 30-35 metres in an ordinary test conditions despite the antenna.

The module itself can't communicate on its own as it requires some sort of encoding before transmitting data and interpreting or decoding once the transmitted signal is received; so it must be utilized with an encoder or decoder integrated circuit (IC) or with any microcontroller at both ends; here we have used the Atmega328 to facilitate that, by mounting it on Arduino board. The least complex approach to utilize it is with the HT12E encoder and HT12D decoder IC [2].

The module utilizes ASK (Amplitude Shift Keying) technique, thus making it simple to interface with microcontrollers. On the off chance that one attempts to utilize this with Arduino, as we have done in this case, at that point the Radiohead library makes things simple for the programmer. However one can't expect information without interference in case of a long distance communication as this module is particularly susceptible to noise. The range relies upon the voltage provided to Receiver and the noise present in the surroundings [3].

\section{Accelerometer}

The ADXL345 is a low-power, 3-axis microelectromechanical system (MEMS) accelerometer module with both Inter-Integrated circuit (I2C) interface and serial peripheral interface (SPI). The sensor comprises of a microscale machined structure on a silicon wafer. The structure is suspended by polysilicon springs which enable it to shift easily toward any path smoothly when subject to increasing speed in the $\mathrm{X}, \mathrm{Y}$ as well as $\mathrm{Z}$ axis. The

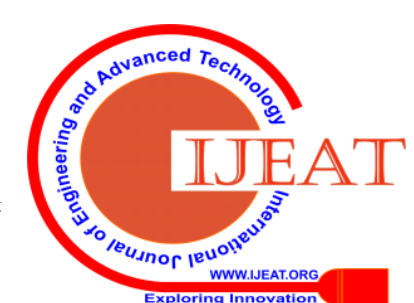


displacement causes a variation in value of capacitance between the fixed plates and the movable plates (which move due to being attached to the suspended structure). This variation in capacitance along every axis is converted in order to yield an output voltage corresponding to the acceleration along respective axes [4].

The ADXL345 Breakout has an I2C address of 0x53. It can share the I2C bus with other I2C gadgets as long as every gadget has a unique address. Mostly, only 4 connections are required for I2C communication: Serial Data (SDA), Serial Clock (SCL), Voltage Common Collector (Vcc) and Ground (GND) [5].

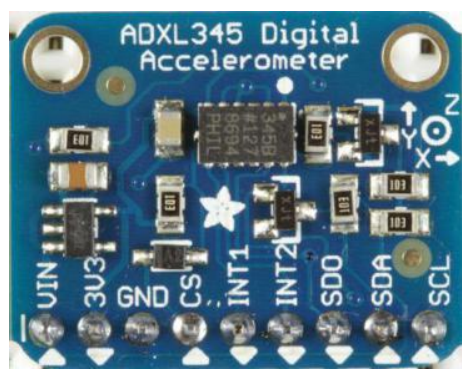

Fig. 3. Accelerometer (ADXL345)

Table- I: Arduino Uno Technical Specifications

\begin{tabular}{|l|l|}
\hline \multicolumn{1}{|c|}{ Microcontroller } & \multicolumn{1}{|c|}{$\begin{array}{c}\text { ATmega328p - 8 bit AVR family } \\
\text { microcontroller }\end{array}$} \\
\hline Operating Voltage & $5 \mathrm{~V}$ \\
\hline Recommended Input Voltage & $7-12 \mathrm{~V}$ \\
\hline Input Voltage Limits & $6-20 \mathrm{~V}$ \\
\hline Analog Input Pins & 6 (A0 - A5) \\
\hline Digital I/O Pins & $\begin{array}{l}14 \text { (Out of which 6 provide PWM } \\
\text { output) }\end{array}$ \\
\hline DC Current on I/O Pins & $40 \mathrm{~mA}$ \\
\hline DC Current on 3.3V Pin & $50 \mathrm{~mA}$ \\
\hline Flash Memory & $32 \mathrm{~KB}(0.5 \mathrm{~KB}$ is used for Bootloader) \\
\hline SRAM & $2 \mathrm{~KB}$ \\
\hline EEPROM & $1 \mathrm{~KB}$ \\
\hline Frequency (Clock Speed) & $16 \mathrm{MHz}$ \\
\hline
\end{tabular}

\section{DC Motors}

Center shaft direct current (DC) motor with gear box is a motor which has good torque and high rpm even at lower voltages and is excellent for attaching to mechanical objects such as wheels to deliver smooth rotary movements. This motor operates at 300rpm when supported by a $5 \mathrm{~V}$ battery, which can be increased up to 500rpm provided its supply is increased to $12 \mathrm{~V}$ [6].

\section{E. Driver Module}

L293D is a motor driver integrated chip and as the name implies, facilitates movement of motor attached to it. It is a 16 pin IC that has the capacity to control two DC motor and the concept is based on H-bridge. However, in order to increase the battery life, we have used two motor driver ICs, attaching only one wheel per motor driver; this also prevents overheating of the IC. For having the capacity to turn, the voltage needs to change it's direction in clock and against clockwise bearing [7].

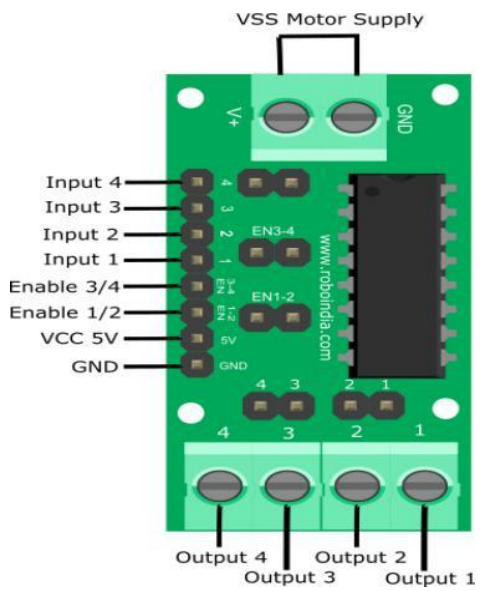

Fig. 4.L293D Driver Module

\section{METHODOLOGY}

\section{A. Approach}

The modus operandi of this project involves beginning with splitting the model into two modules, resulting in two circuits: (a) a transmitter circuit, and (b) a receiver circuit. The methodology behind this is that we will connect the ADXL345 with the Atmega328p IC which will process the values fed to its analog pins from the accelerometer. This data will be sent out through the Arduino Uno board's data pin into the RF433 transmitter which will be responsible for propagating the data to the receiving circuit i.e. the toy car. At the receiving end, the $\mathrm{RF}$ receiver module catches the signal transmitted to it, since it is programmed to operate at the same baud rate as that of the RF Transmitter (9600bps) thus limiting the probability of interference and receiving an incorrect signal. The RF module transmits the data to L293D via the Arduino SMD board. In the present model, two motor driver ICs (L293D) have been used, each connected to a DC motor, which facilitates the rotation of the wheels [8].

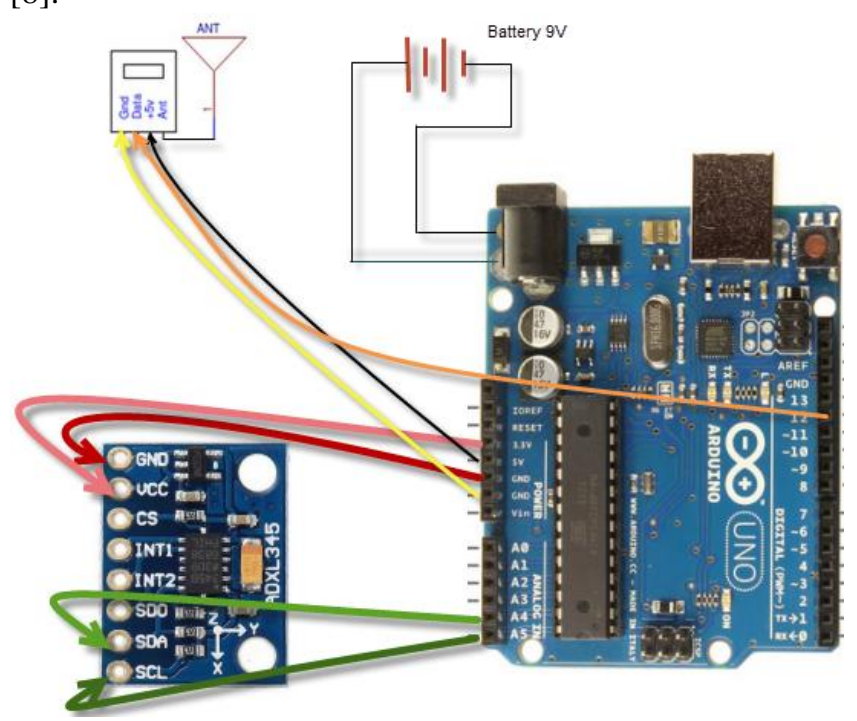

Fig. 5. Transmitting circuit connection diagram.

\section{B. Key Differences}

Some of the key changes in this model from existing ones are:

Published By:

Blue Eyes Intelligence Engineering \& Sciences Publication

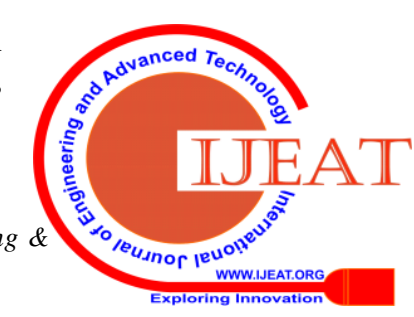




\section{Gesture Controlled Robot using Accelerometer}

- Using slave Arduino UNO in receiver circuit, minimising complexity,

- Using a 3 wheel chassis to reduce size and improve maneuverability,

- Reducing the motor driver to wheel ratio to $1: 1$ to enhance power efficiency.

The receiving circuit has been equipped with another Arduino UNO module for communication ease. Not only does the 328p SMD module improve communication speed, it replaces the need for installing a separate decoder at the receiving end; thus eliminating the need to install an encoder - decoder pair such as HT12E - HT12D ICs. This results in reduced number of connections and thus lesser complexity.

Use of a 3-wheel chassis reduces the robot size, improves maneuverability as well as reduces need for power supply as would've been the requirement in case of 4 wheels. Here only two wheels require power as the robot is modified into a rear-wheel drive car.

Also, to manage power consumption more efficiently, each L293D motor driver module has been connected to only one wheel and only $5 \mathrm{~V}$ power is supplied to the wheels. This provides adequate speed while improving battery life of the model along with preventing overheating of the L29D IC. The adjustment to increase the robot speed requires minor connection changes on the power output pins of the module to $9 / 12 \mathrm{~V}$ from $5 \mathrm{~V}$ that supply power to the $\mathrm{DC}$ motor.

Thus, the increased cost due to the SMD board is nearly compensated by the cost reductions due to the removal of the encoder-decoder pair, smaller chassis, lesser wheels, and by modifications for

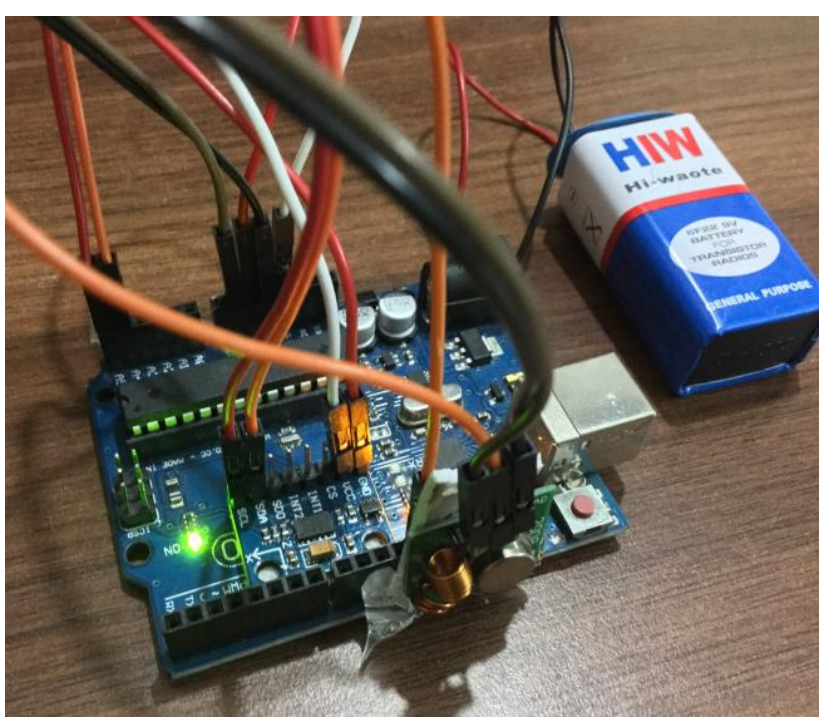

Fig. 6. Transmitting module.

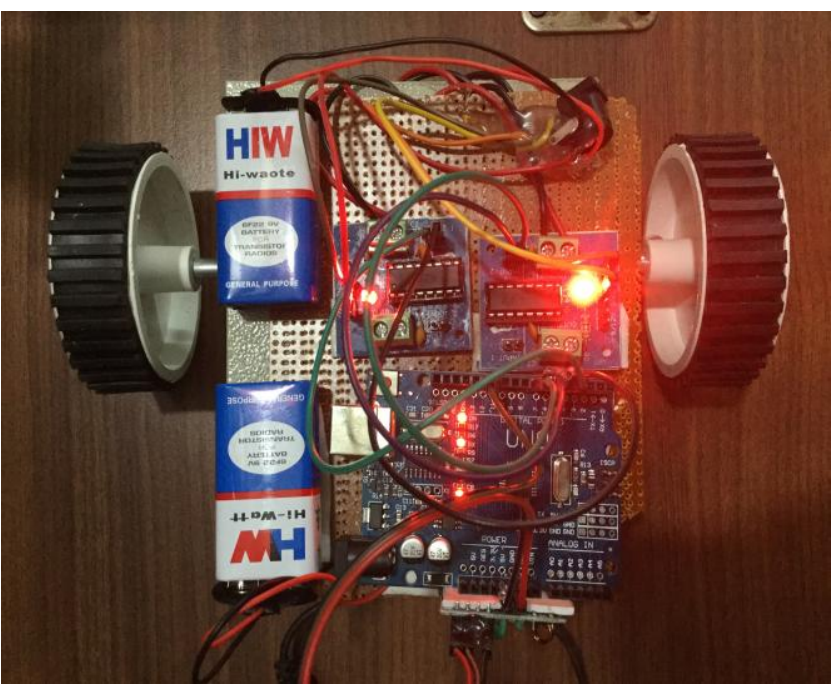

Fig. 7. Receiver module.

\section{CONCLUSION}

We accomplished our goal with no obstacles i.e. controlling a robot with gestures rather than remote controlled devices. Our robot is indicating legitimate reactions at whatever point we move our hand. The output from the four pre-defined hand motions to make the robot move in desired directions are: flexion for forward motion, extension for backward motion, tilt right for right turn and tilt left for left turn.

\section{FUTURE SCOPE}

This model, though suitable for the toys industry as a toy car for kids, has scope for improvements which shall result in its adaptability and expansive utility in varied domains such as for military implementations, exploration usage, in medical field etc. Some of them are:

- replacing RF module with a bluetooth module in order to facilitate communication between the transmitting and receiving circuit,

- giving the model car a pair of eyes by installing a camera for surveillance purposes,

- installing a GPS module to help in mapping hostile terrains,

- equipping the model with a solar battery to replace the primary power supply, and falling back on the mounted Li-Ion batteries as back-up only in case of emergency.

\section{REFERENCES}

1. Arduino, "ARDUINO UNO REV3,"

(2018) Available: https://store.arduino.cc/usa/arduino-uno-rev3 M. Rawashdeh, "Food Living Outside Play Technolog RF 315/433 MHz Transmitter-receiver Module and Arduino," (2013).

3. "ASK Super Regenerative Receiver ST-RX02-ASK Receiver General Description: Applications Car security system Wireless security systems Sensor reporting Automation system Remote Keyless entry,' 2006.

4. Analog Devices, “ADXL345 Rev. E.” pp. 13. Available: http://www.analog.com

5. "ADXL345 Digital Accelerometer," 2019

6. S.K. Bhattacharya, Basic Electrical and Electronics Engineering (2nd ed.) 2016, ch. 6.

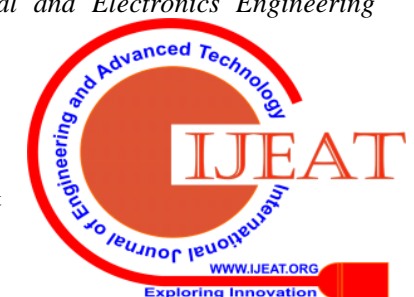


7. Texas Instrument, "L293, 1293d quadruple half-h drivers," Office, 2002. Available: http://users.ece.utexas.edu/ valvano/Datasheets/L293d.pdf

8. A. Nath, P. Chanda, K. Mukherjee, and S. Modak, "Gesture Controlled Robot using Arduino and Android Authenticating and Authorising Digital Transcripts using QR Code View project Detection of Different Stages of Lungs Cancer in CT-Scan Images using Image Processing Techniques View project Gesture Controlled Robot using Arduino and Android," 2016.

\section{AUTHORS PROFILE}

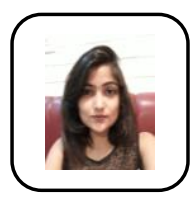

Aishwarya Mohan completed her B.Tech degree in Electronics and Communication Engineering from Sharda University in 2019. She has worked as a Performance testing engineer, is an NCC C certificate holder, received Certificate of Distinction in Quadrotor building issued by Skifi Labs, and is proceeding for her Masters degree this year. Her areas of interest are Wireless Systems, Interactive Technology and Sustainable Development.

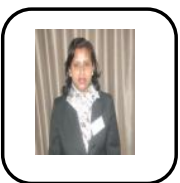

Dr. Rashmi Priyadarshini received her Doctorate and her Master of Engineering degree in Electronics and Communication Engineering from IGIT, GGSIP University. Her Bachelors degree is from GradIETE, New Delhi, India.

With a total experience of over 14 years in teaching and research, she has over 35 research papers published. She is an Associate Professor in Sharda University in the Department of Electronics and Communication. She specializes in Microcontrollers \& Microprocessors with areas of interest being Wireless Sensor Networks, and Embedded Systems.

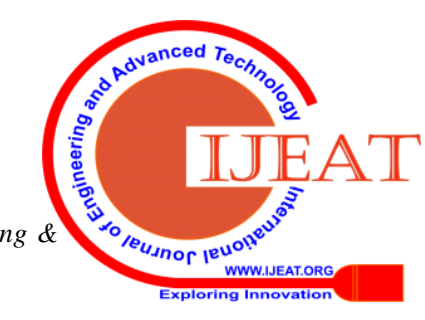

